\title{
Explaining Modern Chinese Women's Views on BM Style Clothes: From Personal Aesthetics and Social Gender
}

\author{
Shijie Wang ${ }^{1, *}$ \\ ${ }^{1}$ University of Connecticut, Storrs, Connecticut, United States \\ *Corresponding author. Email:shijie.3.wang@uconn.edu
}

\begin{abstract}
In recent years (approximately from 2019-2021), BM-style clothes have become very popular among young Chinese women. BM is the acronym of Brandy Melville. It is a European clothing and fashion accessories brand, which markets its products to young women. The company was established in Italy by Silvio Marsan but attracted most of its popularity in California. The characteristics of BM-style clothes are short, tight, and exposed. BM style clothes also represent "S size girl". The sizes of all clothes are mainly available in S or small sizes. So, what makes this style of clothes so popular in China? This article will explore the attitudes of contemporary Chinese young women towards BM-style clothes through a questionnaire. Through the form of questionnaires, this research can be made more objective, uniform, and extensive. The article will explore and find the answer to the influence of social media, social gender, and personal aesthetics on women's clothing. Does the popularity of BM-style clothes have anything to do with the low price and the exposure rate on social media? The research aims to hope that the government calls on the masses' health awareness and women will stop blindly pursuing unhealthy figures and meaningless dieting. However, this article only studies the influence of BM-style clothes in China, and there may be a singularity. In the future, it can be compared with related articles in other countries or regions to better understand how BM-style clothes are spread globally through personal aesthetics and social media.
\end{abstract}

Keywords: Chinese Society, Young Women, BM Clothes, Communication, Appearance Anxiety, Body Shame

\section{INTRODUCTION}

The essay's research question is why BM clothes are so popular in China. From 2017 onwards, this style was getting popular in China. However, the phenomenon is under-researched. This question is complex and unique because there are many reasons behind it. I will try to answer this question through the survey method. BM clothes originated from European countries, became popular in the US, and finally spread to China. The essay will focus on BM clothes in the case of China because Chinese people have a different understanding and perception of beauty from the US and UK people. This understanding of beauty has an impact on the popularity of BM clothes. This research can be applied to other countries in East Asia because they also have a relatively similar understanding of beauty and fashion. In other words, this essay will have broader implications.
This research is important because it can deepen our understanding of why BM clothes have become so popular in China and why so many Chinese youth girls like to buy Bm clothes. Moreover, it explores the deep reason in the social understanding of beauty from a sociological perspective. Also, I aim to explain this from the aspect of social media.

The essay is divided into several sections. After the introduction, I will discuss the origin of BM clothes and how BM became popular in China. In the next section, I will look into the explanations from the economic aspect, the social media aspect, and the sociology aspect. Then I will describe the date and method of document analysis and survey research. After that, I will analyse the survey result in relation to the theory. The final conclusion provides directions for further study. 


\section{THE RISE OF BM STYLE CLOTHES AND THEIR SPREAD IN THE WORLD AND CHINA}

\subsection{The Origin of BM Cloths}

The full name of BM is Brandy Melville, a women's clothing brand from Italy. The company was founded in the early 1990s by father and son Silvio and Stephan Marsan. But the place that really makes it worthwhile is Californian, North America. The BM brand quickly developed in the United States and is very popular among teenagers. Many young and thin girls choose BM because these brands are cheap and good-looking. Compared with the well-known clothing brands such as Uniqlo and ZARA, BM is closer to the aesthetics of the student in style. The most common style elements are plaid, floral, camisole, and knitted short top. Brandy Melville's brand image is constructed exclusively through online platforms, and the fame is largely due to the company's effective use of social media. The brand's primary medium is Instagram, where the official, USA- based account @ brandymelvilleusa currently has more than 3.5 million followers (August 2021). Most of the images feature young, slender, longhaired models, so-called Brandy Girls, displaying the outfits that emanate an easy-going Californian lifestyle. In addition to the brand's innovative social media strategy, Brandy Melville stands out from the crowd with a distinctive one size fits most policy, aiming to make shopping easier because all the pieces come in one size. However, most of the one-sized clothes are equal to a size small - a size which does not fit most - which makes the brand's slogan quite controversial. According to Emilia Mansikka, the sizing policy has raised discussion and concern in the media and over the internet on topics such as fat-shaming, discrimination, and young girls' self-esteem [1].

\subsection{BM Style In China}

BM style clothes originated in Europe, rise in the United States, and then introduced to Asia. According to Emilia Mansikka's research, "Nowadays Brandy Melville has some 30 stores in the United States, most of which are located in California, nine stores in Canada, nearly 40 stores all around Europe, and very recently the brand has even expanded to Asia, opening three stores in Singapore and Bangkok (Brandy Melville 2017) altogether.'[1] I found that there is no appearance in China, which is a research gap. So, in this section, I will explore the development of BM-style clothes in China.

According to sjfzxm.com, in 2019, BM officially settled in mainland China and opened its first store in Shanghai. There are a large number of customers in the store during business hours, and the changing rooms are often lined up [2].

\section{EXPLAINING THE POPULARITY OF BM STYLE CLOTHE IN CHINA}

In this section, I will explain the popularity of BM style clothes in China from the following four perspectives: Price factor, Social Media app, Social aesthetics, and Personal aesthetics.

\subsection{Price Factor}

Price plays an important role in affecting buying behavior of customers. Safitri argues that the higher the price of the clothes, the more like people will buy them [3]. This is because people tend to think more expensive clothes are of high quality. However, I do not agree with this idea. Because it depends on the purchasing power of consumers, I argue that one of the important reasons for the popularity of BM clothes is that it has an affordable price. In other words, BM clothes are not luxurious, and young Chinese girls who just stepped into society would still be able to buy such clothes. For young women in China, they may not have a high enough salary to buy expensive clothes. Therefore, I propose the following hypothesis:

H1: Chinese young women prefer to buy less expensive clothes.

\subsection{Social Media App}

Besides the price factor, another important reason for the popularity of BM clothes is social media apps. According to Marsh Lisa, In foreign countries, Instagram and Twitter play an important role in the popularity of BM clothes [4]. This explanation is powerful because social media has played a dominant role in our lives, and people spend a large amount of time using social media. When young girls post pictures of themselves wearing BM clothes on social media and gain many 'likes', the BM fashion can gradually become a fashion in society. Also, many companies may use social media as their marketing strategy to promote BM clothes. However, this explanation is limited because, in China, Instagram and Twitter are not commonly used. Instead, the social media that young women in China prefer to use are Xiaohongshu, Weibo, etc. The more posts and views on social media, the greater the probability of young Chinese women purchasing. Therefore, I propose the following hypothesis:

H2: The more Social Apps people use, the more frequently they will wear BM-style clothes.

\subsection{Social Aesthetics}


Each country has a different perception of beauty. According to the information released by IPSOS in 2019, China does not think of humor and Intelligence as beautiful [5]. On the other hand, in terms of body weight and shape, with an average score of 6.4 , China scores 7.8, ahead of all countries. A major feature of BM-style clothes is that they only sell small size clothes, which undoubtedly makes BM more popular in China. Therefore, I propose the following hypothesis:

H3: In China, popular aesthetics have an impact on the purchase rate of BM style clothes

\subsection{Personal Aesthetics}

According to the previous section, China's public aesthetics itself tends to be thin for beauty. With the advancement of technology, weight loss tweets on various social media and the Internet are also stimulating people and causing women's appearance anxiety. Also, according to a BBC report, many people believe "Being slim and beautiful implies 'good value' as an object" [6]. In such an environment, the personal aesthetics of young Chinese women are also biased towards slim figures. Therefore, I propose the following hypothesis:

H4: Personal aesthetics has an impact on the purchase rate of $B M$ style clothes

\section{RESEARCH DESIGN}

In this section, I will describe the two methods that I will use. The first is document analysis. I will analyze the documents in social media and from the official website of BM clothes. This is because document analysis can provide us with reliable and authentic information. I will also triangulate document analysis with survey research. This is because survey research can inform us of the opinions of young people in Chinese society. I am aware that both methods have their strengths and limitations. However, using a basket of methods could effectively reduce research bias and improve the research rigour. In this section, I will analyse the strengths and limitations of the two methods. I will also provide definitions of the two methods. Moreover, I will present how I use these two methods to operationalize the research.

\subsection{Document Analysis}

According to Bowen, document analysis is 'a systematic procedure for reviewing or evaluating documents - both printed and electronic (computer-based and Internet-transmitted) material.' Moreover, Bowen shows that the procedure for document analysis includes 'skimming (superficial examination), reading (thorough examination), and interpretation' [7].
Strauss and Corbin demonstrate that the purpose of the interpretation is 'elicit meaning, gain understanding, and develop empirical knowledge' [8].

In my research, I set up questionnaires and document analysis, consisting of official website data and articles on social media. Social media include Xiaohongshu, Weibo, and Bilibili. The advantage of text analysis lies in its easy availability, non-confidentiality, and can be obtained in large quantities. Stake reveals that the advantage of document analysis is that it can provide 'rich descriptions of a single phenomenon, event, organization' [9].

Furthermore, document analysis can also be combined with other methods to analyze problems. Denzin suggests that' document analysis is often used in combination with other qualitative research methods as a means of triangulation - the combination of methodologies in studying the same phenomenon' [10].

But at the same time, document analysis also has its shortcomings. Compared with the questionnaire, document analysis' data and choices do not fail to meet my research project, and there is a certain amount of data bias in document analysis.

Yin shows that document analysis suffers from the weakness of 'biased selectivity'. According to Yin, biased selectivity means that 'In an organizational context, the available (selected) documents are likely to be aligned with corporate policies and procedures and with the agenda of the organization's principles' [11].

\subsection{Survey Research}

My questionnaire design time is between July 3 and July 10, 2021. The questionnaire is released on July 10th and ends on July 18. The main respondents to my questionnaire came from the WJX (Questionnaire Star) and WeChat. I chose WJX as the platform for publishing questionnaires. This is an online questionnaire platform. The reason for my choice is that it is simple to operate, has many functions, and is used by many users on the website, with more than 20 million users. Because this is the first time I have released a questionnaire, I am worried that I lack experience and will get invalid questionnaires or an insufficient number of questionnaires. So, in the subsequent questionnaire settings, for some sensitive questions, I set options that can be avoided and use more euphemistic language, such as a question about weight loss. Questionnaires can be obtained on request.

\section{ANALYSIS}

This section will use the survey results to analyze why BM-style clothes are popular in China. The analysis will be conducted in the following four aspects: 
Price factor, social media App, social aesthetics, and personal aesthetics

\subsection{Price Factor}

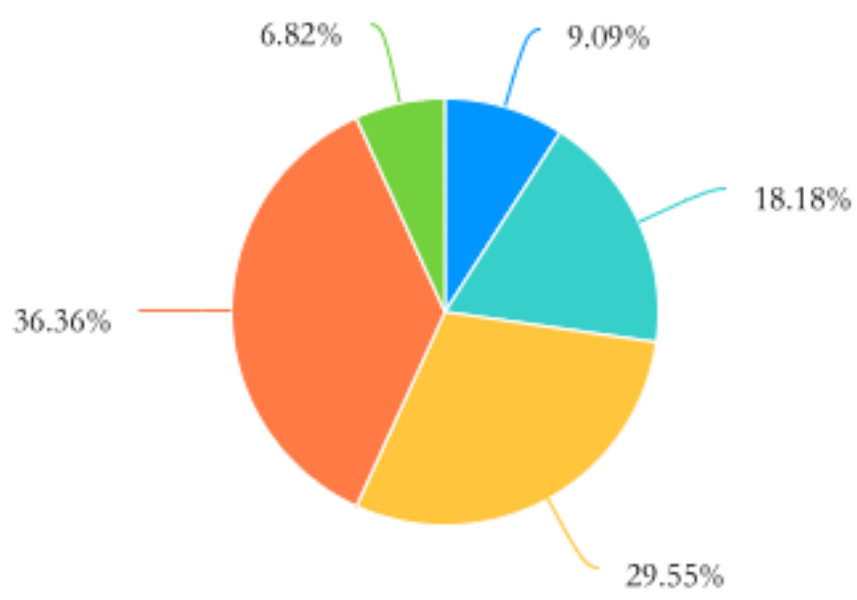

Figure 1. Price factor

Figure 1 shows the frequency of Buying BM-style clothes for Chinese youth women. From Fig 1, it's clear that they will buy BM-style clothes between every season and every half year. $9.09 \%$ of the respondents express that they will buy it every week. Hence, it shows that the price of $\mathrm{Bm}$ clothes is affordable to Chinese youth women. Therefore, the H1 is proven:

H1: Chinese young women tend to buy less expensive clothes.

\subsection{Social Media App}

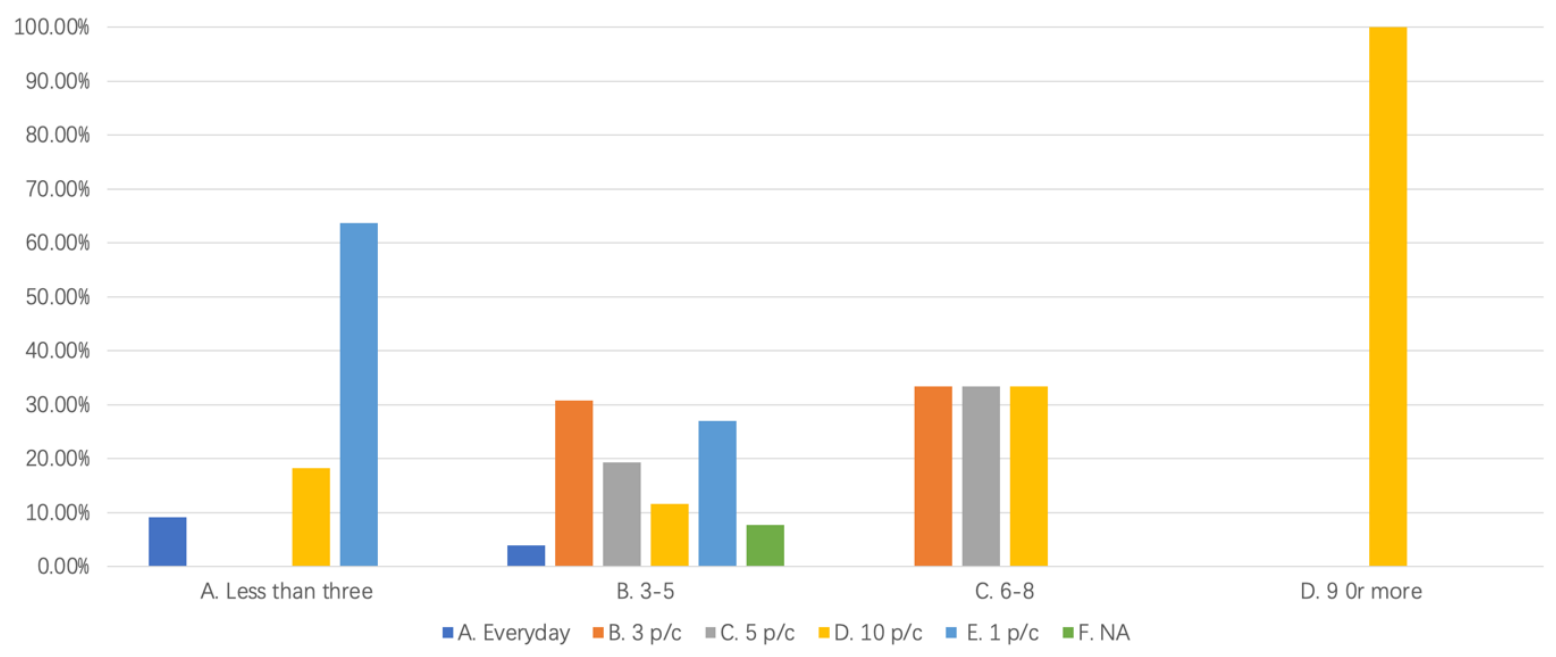

Figure 2. Social Media App and Wear frequency

Figure 2 shows the number of Chinese young women using social media and how often they wear BM-style clothes. In the figure, we can see that when the number of social media used by young women is less than three, the frequency of wearing BM-style clothes is equal to or less than once a month. But when the number of social media they use increases to 3-5, their frequency of dressing will increase to once every 3-4 days. Therefore, the $\mathrm{H} 2$ is proven: 
H2: The more Social Apps people use, the more frequently they will wear Bm style clothes.

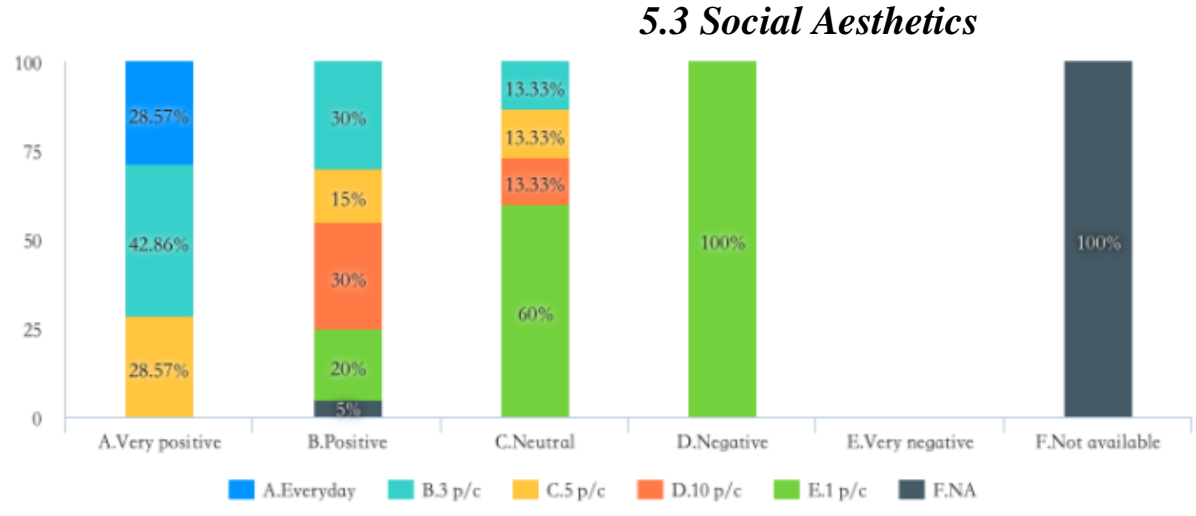

Figure 3. Social aesthetics and wear frequency

Figure 3 shows that when people around you show a positive attitude about BM clothes, you will be more likely to wear them every day. The data shows that $28.57 \%$ of the respondents wear BM clothes every day. For the people who wear it three times per month, $42.86 \%$ of people around have a very positive attitude, and $30 \%$ of the people around have a positive attitude.

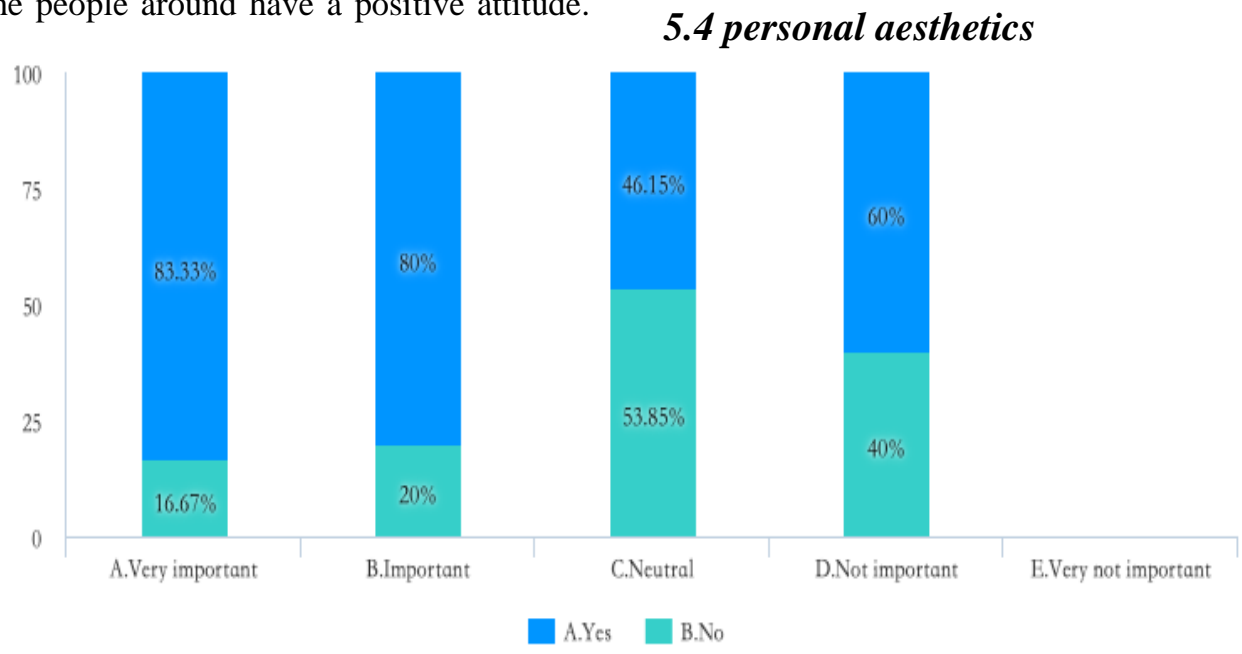

Figure 4. Personal aesthetics and Attitude of losing weight

Figure 4 demonstrates that if the respondents think that losing weight is important, then he or she will be more likely to be anxious about appearance. For example, for the people who think losing weight is very important, $83.33 \%$ of them have appearance anxiety. While for those people who think losing weight is neutral or not important, only around half of the people have anxious feelings about their appearance. Hence, the following $\mathrm{H} 4$ is confirmed:

H4: Personal aesthetics has an impact on the purchase rate of BM style clothes
On the other hand, most of the people around them hold neutral and negative attitudes for those who wear one time per month. Therefore, the $\mathrm{H} 3$ below is proven:

H3: In China, popular aesthetics has an impact on the purchase rate of BM style clothes

\section{4 personal aesthetics}

\section{CONCLUSION}

In summary, the findings of this research show that there are many reasons for the popularity of BM clothes in China. The analysis shows that an affordable price is important, while the root reason is social aesthetics. When the whole society considers being slim is beautiful, this has an impact on personal aesthetics. Such an attitude has spread across various social media platforms and makes Chinese youth women buy BM clothes.

This research deepens our understanding of social psychology about beauty and also the impact of social media. Finally, this research contributes to the study of fashions. In terms of policy implications, this essay can 
recommend the Chinese government to make policies to lead social opinions about beauty. Also, this assay can provide references to companies when making marketing strategies. So they can better use social media to promote their clothes.

The essay has several limitations. The analysis may be incomplete, and maybe there are other reasons. Also, the sample of this research is limited. The single case provides limited explanations and may not fully explain other cases. Further studies can look into the popularity in other countries, such as American and European countries.

\section{REFERENCES}

[1] E. Mansikka, (2019). One size fits most? -Visual Representation of Women in Brandy Melville's Instagram Images.

[2] sjfzxm.com, 2020. "BM Girl" Sweeps Across The City Beauty, Who Is Boosting "Sweet, Spicy And Thin"? Retrieved from: http://www.sjfzxm.com/global/en/570415.html

[3] I. Safitri, (2018). The Influence of Product Price on Consumers' Purchasing Decisions. Review of Integrative Business and Economics Research, 7, 328-337.

[4] L. Marsh, (2014) “Brandy Melville: Instagram's First Retail Success." Bloomberg Businessweek. Retrieved from: https://www.bloomberg.com/bw/articles/2014-12-1 1/brandy-melville-instagram-s-first-retailsuccess

[5] IPSOS, 2019. Retrieved from: https://www.ipsos.com/sites/default/files/ct/news/d ocuments/2019-08/global-advisor-beauty-august-2 019.pdf

[6] BBC, 2021.'China's latest online skinny fad sparks concern' Retrieved from: https://www.bbc.com/news/world-asia-china-5634 3081

[7] G. A. Bowen, (2009). Document analysis as a qualitative research method. Qualitative research journal.

[8] A. Strauss \& J. Corbin, (1998). Basics of qualitative research techniques (pp. 1-312). Thousand oaks, CA: Sage publications.

[9] R. E.Stake, (1995). The art of case study research. sage.

[10] N. K. Denzin, (2017). The research act: A theoretical introduction to sociological methods. Transaction publishers.
[11] R. K.Yin, (1994). Case study research: Design and methods (2nd ed.). Thousand Oaks, CA: Sage. 